\title{
Étude du niveau de production larvaire d'Anopheles gambiae s.I. (Diptera: Culicidae) dans différents types de gîtes à Oussou-yaokro au Centre-Ouest et à Korhogo, au Nord (Côte d'Ivoire).
}

$\mathrm{Tia}^{1{ }^{*} E m m a n u e l, ~ G b a l e ́ g b a}{ }^{2,3}$ N.G. Constant, M'Bra ${ }^{2,4}{ }^{4}$. Richard, Kaba2,5Aboudramane, Boby' ${ }^{1}$ O.A Marie, Koné1 Moussa, Chouaibou² Mouhamadou, Koné2,6 Brama, Koudou²,3,7 G. Benjamin

1. Centre d'Entomologie Médicale et Vétérinaire, Université Alassane Ouattara, Côte d'Ivoire, 27 BP 529 Abidjan 27

2. Centre Suisse de Recherches Scientifiques (CSRS) en Côte d'Ivoire, 01 BP 1303 Abidjan 01, Côte d'Ivoire.

3. UFR des Sciences de la nature, Université Nangui Abrogoua, 02 BP 801 Abidjan 02, Côte d'Ivoire

4. UFR des Sciences de la Terre et des Ressources Minières, Université Félix Houphouët Boigny, 22 B.P. 582,

Abidjan 22, Côte d'Ivoire

5. 5. UFR de Biosciences, Université Félix Houphouët Boigny, 22 B.P. 582, Abidjan 22, Côte d'Ivoire

6. Université Péléforo Gon Coulibaly, BP 1328, Korhogo, Côte d'Ivoire.

7. Liverpool School of Tropical Medicine, Pembroke Place, Liverpool L3 5QA, UK.

*Auteur correspondant:" Tia Emmanuel: emtia1fr@yahoo.fr, cel (+225) 06533870

Original submitted in on $3^{\text {rd }}$ August 2016. Published online at www.m.elewa.org on 30th September 2016

http://dx.doi.org/10.4314/jab.v105i1.13

\section{RESUME}

Objectif : Le paludisme est un problème de santé publique en Côte d'Ivoire. La lutte contre les vecteurs de cette affection nécessite la connaissance de leur lieu de reproduction. Cette étude se propose d'identifier les gites habituels et éventuellement les gîtes inhabituels (ou atypiques) d'An. gambiae s.l. en milieu rurale et en milieu urbain.

Méthodologie et résultats : Des prospections larvaires dans divers points d'eau et la mesure des paramètres physico-chimiques ont été menées à Oussou-yaokro (milieu rural) pendant 4 mois et de manière transversale à Korhogo (milieu urbain). A Oussou-yaokro, 41 gîtes ont été recensés contre 44 à Korhogo et repartis en gîtes classiques et en gîtes atypiques (non habituels) d'An. gambiae s.l. Les gîtes atypiques sont par ordre d'importance les citernes ou jarres de conservation domestique d'eau, les abreuvoirs d'animaux, les eaux usées de fabrication de beurre de Karité, fosses septiques et des pneus usagés. La majorité des gîtes positifs se trouvent dans l'environnement immédiat des populations locales. A Oussou-yaokro, les gîtes atypiques produisent de manière similaire les larves que les gîtes classiques contrairement à Korhogo où les gîtes habituels sont plus productifs que les gîtes atypiques. La dynamique des gîtes a montré que les jarres ont une production larvaire régulière sur toute l'étude aussi bien en saison sèche qu'en saison des pluies en milieu rural. Quant à la productivité des gîtes, elle est liée à la pluviométrie en saison des pluies, mais aussi aux habitudes et à la durée de conservation d'eau dans les récipients en saison sèche. 
Tia et al. J. Appl. Biosci. 2016 Étude du niveau de production larvaire d'Anopheles gambiae s.l. dans différents types de gîtes à Oussou-yaokro au Centre-Ouest et à Korhogo Côte d'Ivoire.

Conclusion et application des résultats : La présente étude indique qu'An. gambiae s.l. se développe aussi dans des gîtes atypiques, tels que les jarres, les abreuvoirs d'animaux, les eaux des fosses septiques, les eaux résiduelles de beurre de karité, initialement propices à la reproduction d'Aedes aegypti et de Culex quinquefasciatus. Au regard de nos résultats, la lutte antivectorielle contre les vecteurs du paludisme doit prendre en compte tous les points d'eau sans exclusive en raison de l'ubiquité d'An. gambiae s.l.

Mots-clefs : An. gambiae s.I., production larvaire, type de gîtes, milieu rural, milieu urbain, Oussou-yaokro, Korhogo, Côte d'Ivoire

Study of Anopheles gambiae s.l. ( Diptera culicidae) larval production level in different types of breeding sites in Oussou-yaokro, West-Central and in Korhogo, Northern (Côte d'Ivoire)

\section{ABSTRACT}

Objective: Malaria is a public health problem in Côte d'Ivoire. The control of the vectors requires knowledge of their breeding sites. This study aims to identify the usual and the possibly unusual (or atypical) breeding sites of An. gambiae s.I. in rural and urban areas.

Methods and Results: Larval surveys in various water points and measurement of physicochemical parameters were carried during 4 months in Oussou-yaokro (rural area) and by cross-sectional study in Korhogo (urban area). Forty one (41) and 44 sites were identified in Oussou-yaokro and Korhogo respectively and divided into classic and atypical (not usual) breeding sites of An. gambiae s.l. Atypical breeding habitats were in order of importance, tanks or jars for domestic water conservation, drinking troughs for animals, waste water due to shea butter manufacturing, septic tanks and used tires. Most of positive breeding sites were in the immediate environment of local populations. In Oussou-yaokro, the abundance of larvae in atypical breeding sites and in usual breeding sites was similar while in Korhogo usual breeding sites were more productive. The dynamics of the larvae habitats showed that, in rural area, jars have a regular larval production throughout dry and rainy season. The number of larvae habitats in both localities was related to rainfall during the rainy season, but also to practices of population and period of water retention in the containers.

Conclusion and application of results: The present study indicates that An. gambiae sl. can also breed in unusual habitats, such as jars, animal drinking troughs, septic tanks, wastewater of shea butter, initially suitable for breeding of Aedes aegypti and Culex quinquefasciatus. In light of these results, vector control against malaria vectors must consider all water points without exception because of the ubiquity of $A n$. gambiae s.l.

Keywords: An. gambiae s.l., larval production, breeding sites, rural area, urban area, Oussou-Yaokro, Korhogo, Côte d'Ivoire

\section{INTRODUCTION}

Le paludisme constitue aujourd'hui un réel problème de santé publique qui menace plus d'un milliard de personnes vivant dans la ceinture de pauvreté (OMS, 2014a). On estime à 247 millions le nombre de cas de paludisme sur 3,3 milliards de personnes à risque en 2006 ; environ 665000 de ces cas sont mortels, dont environ $90 \%$ surviennent principalement en Afrique subsaharienne (OMS, 2015). En Côte d'Ivoire, le paludisme représente la première cause de mortalité (en occasionnant 33\% des décès), de consultation ( $60 \%$ des motifs de consultations) et d'hospitalisation $(68 \%$ des hospitalisations) dans les formations sanitaires; il est en outre responsable de la perte de $52 \%$ de la production agricole et $40 \%$ d'absentéisme (OMS, 2014b).Cette pathologie est transmise à l'homme par l'intermédiaire de vecteur du genre Anopheles. Ces vecteurs se reproduisent dans des points d'eau dont les caractéristiques physiques et chimiques classiques ont été décrits; par exemple, Anopheles gambiae s.l. vecteur majeur du paludisme, se développe, selon plusieurs auteurs dans des points 


\section{Tia et al. J. Appl. Biosci. 2016 Étude du niveau de production larvaire d'Anopheles gambiae s.l. dans}

différents types de gîtes à Oussou-yaokro au Centre-Ouest et à Korhogo Côte d'Ivoire.

d'eau ensoleillés, claire et propres. Quant à $A n$. funestus s.l., il est connu inféodé aux gîtes ombragés par des végétaux aquatiques (Mouchet et al., 2004). Outre ces gîtes classiques, les populations créent de plus en plus dans leur environnement immédiat d'autres points d'eau soit par leurs habitudes de conservation d'eau dans des jarres ou par des activités économiques telles que la transformation artisanal de plantes locales telles que le karité dont l'extraction du beurre occasionne les eaux résiduelles recueillies dans des jarres. Les jarres d'eau ou des eaux résiduelles de fabrication de beurre de karité sont connus être propices au développement des espèces d'Aedes vecteurs de la fièvre jaune ou de Culex quinquefasciatus (Yebakima, 1989 ; Girod et Le Goff, 2006). En revanche, selon des auteurs, la bio écologie

\section{MATERIEL ET METHODES D'ETUDE}

Présentation des sites d'étude : L'étude a été menée de manière longitudinale à Oussou-yaokro $\left(5^{\circ} 49^{\prime} 50^{\prime \prime}-\right.$ $5^{\circ} 44^{\prime} 45^{\prime \prime} \mathrm{L} \mathrm{N}$ et $6^{\circ} 55^{\prime} 55^{\prime \prime}-7^{\circ} 1^{\prime} 00^{\prime \prime} \mathrm{L} 0$ ), un village de 602 habitants (INS, 2014) situé dans la préfecture de Bouaflé et de manière transversale dans la ville de Korhogo et ce, pour le recueil d'informations complémentaires relatives au milieu urbain. Oussou-yaokro appartient au secteur pré-forestier qui est une zone de transition climatique de type baoulé en caractérisé par deux saisons sèches (décembre à février et juillet à août) et deux saisons pluvieuses (mars à juin et septembre à novembre (Figure 1). Les pluviométries annuelles varient de 1200 à 1500 $\mathrm{mm}$ (ANADER, 2010). Ce village est situé à $100 \mathrm{~m}$ du fleuve Marahoué et est entouré de bas-fonds utilisés quelques fois à la culture du riz. Ce village a été choisi pour cette étude, entre autres du fait qu'en saison sèche, la population se ravitaille en eau au niveau de la rizière ou du bas-fond et pour sa richesse en divers types de gîtes de culicidés. En outre, les habitants stockent l'eau dans les citernes qu'ils utilisent aussi bien pour les besoins domestiques que pour la boisson. Dans le village d'Oussou-yaokro, la transmission du paludisme est permanente toute l'année. Le paludisme est la première cause de consultation dans le district sanitaire de Bouaflé où l'incidence du paludisme est de 92 pour 1000au cours de l'année 2012 selon le Médecin-Chef du District sanitaire de Bouaflé. La ville de Korhogo $\left(9^{\circ} 29^{\prime} \mathrm{N} 5^{\circ} 36^{\prime}\right.$ 09.49, -5.6) est située au Nord de la Côte d'Ivoire de la Région des Savanes (Figure 1).Le climat est de type soudano-guinéen caractérisé par deux grandes saisons. classique des vecteurs peut être influencée par les changements climatiques au point où on peut les voir se reproduire dans des gîtes inhabituels, et à de nouveaux lieux de repos (Awolola et al., 2007; Coffinet et al., 2009).Par conséquent, une bonne connaissance de la bio-écologie des vecteurs permettrait de mieux orienter les actions de lutte (Koudou et al.,2005, Fournet et al., 2010). L'objectif de cette étude a été de rechercher la présence d'An.gambiae s.l. dans tous les points d'eau rencontrés dans l'environnement humain en milieu urbain et en milieu rural afin d'en identifier des gîtes atypiques de vecteurs du paludisme. L'identification de gîtes atypique permettrait d'améliorer les stratégies de prévention du paludisme par l'éducation sanitaire pour un changement de comportement.

La grande saison sèche (octobre-mai) précède la saison des pluies (Juin-Septembre) marquée par deux pics pluviométriques, l'un en juin et l'autre en septembre. La pluviométrie varie de 1200 à $1400 \mathrm{~mm}$. La végétation du département est celle de la savane arborée, caractérisée par des arbustes et des arbres tels que le Karité (Vitellaria paradoxa) dont est extrait le beurre de karité très utilisé localement à des fins alimentaires, médicinales(maladies de la peau et pulmonaire, etc.) et cosmétiques (Anonyme, 2015). Le choix de cette ville est motivée par le fait qu'elle abrite une intense activité de maraîchage et d'extraction de beurre de karité occasionnant de nombreux points d'eau et comporte des quartiers à potentiel élevé de gîtes larvaires classiques (maraîchers, rizières, flaques) de An. gambiae s.l. et de points d'eau pollués tels que les eaux usées de fausses septiques, les effluents résiduels de beurre de karité, les sténogîtes de crevasses de rochers etc. Les quartiers répondant à ces critères ont été Zone industrielle, Natiokobadara, Dem et Cocody (quartiers insalubres), et Zone industrielle, Mongaha, Kassirimé (quartiers salubres). Concernant particulièrement Natiokobadara, chaque cour constitue une "usine» artisanale de fabrication de beurre de karité où l'on peut voir de grands canaris contenant des eaux noirâtres issues de l'extraction du beurre de karité (financé par le PNUD) et de grosses boules entassées un peu partout. L'extraction artisanale du beurre de karité occasionne une importante consommation d'eau qui est ensuite rejetée sous forme d'effluents liquides très chargés de manières organiques 

différents types de gîtes à Oussou-yaokro au Centre-Ouest et à Korhogo Côte d'Ivoire.

et versés à même le sol ou recueillis dans des récipients ou jarres d'eau résiduelles de fabrication de beurre de karité. A Korhogo, la saison de transmission du paludisme oscille entre 6 et 8 mois (Koffi et al., 2015).

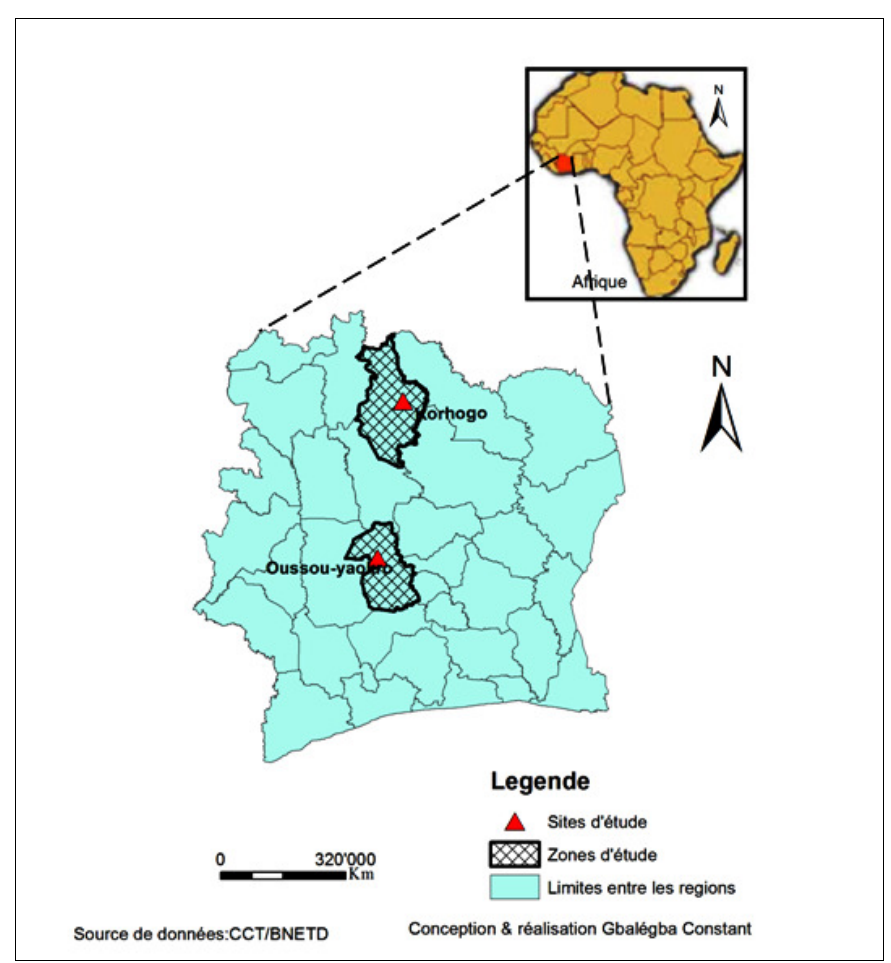

Figure 1: Situation géographique des sites d'étude

Échantillonnage des larves d'An. gambiae s.l. : A Oussou-yaokro, les prospections larvaires ont été menées dans tous les points d'eau du village et alentours dans un rayon de $100 \mathrm{~m}$. A Korhogo, en saison des pluies, les prospections ont été réalisées suivant quatre transects perpendiculaires (en fonction de l'étendue du quartier) tracés dans chaque quartier et à $100 \mathrm{~m}$ de ceuxci. En saison sèche, les gîtes ont été prospectés de manière exhaustive. Les gîtes ont été décrits et leurs coordonnées géographiques ont été relevées à l'aide d'un GPS de la marque Garmin. Les prospections larvaires ont été réalisées une fois par mois à Oussouyaokro de Septembre 2011 à Janvier 2012 et de manière transversale en Juin 2014 (saison des pluies) et mars 2015 (Saison sèche) à Korhogo. La méthode de dipping (Service 1989) à été utilisée dans des gîtes classiques des vecteurs du paludisme et autres points d'eau rencontrés (jarres de conservation d'eau, récipients d'eau résiduelles de karité, fosses septiques). Les larves et nymphes ont été par ladite méthode pendant $5 \mathrm{mn}$ à raison de 20 coups de louche par gîtes dans les gîtes à quantité d'eau suffisante. En ce qui concerne les gîtes de très petites tailles, leur contenu en eau a été entièrement recueilli à l'aide de la louche. Les larves et les nymphes prélevées ont été comptées et ont été soit immédiatement identifiées sur la base des caractères morphologiques, soit après élevage. Tout gîte comportant au moins une larve ou une nymphe d'An. gambiae s.l. est considéré comme positif. A Korhogo, les propriétés physico-chimiques (conductivité en $\mu \mathrm{s} / \mathrm{cm}$, la turbidité en $\mathrm{ppm}$, le $\mathrm{pH}$, oxygène dissous ou $\mathrm{OD}$, la température) de ces gîtes ont été mesurées in situ à l'aide d'un multi paramètre WTW multi350i. A Oussou-yaokro, ces propriétés physico-chimiques n'ont pas été évaluées faute de matériel de mesure lors de l'étude dans cette localité en raison du retard de sa livraison. La production larvaire des gîtes dans les sites d'étude a été déterminée en faisant la somme des larves prélevées dans les gîtes locaux et ce, du fait de la standardisation du temps de prélèvements et de la méthode de dipping (OMS, 1975). Analyse des données : Les données ont été saisies à l'aide du logiciel Excel et analysées par le logiciel Stata version 14.1 (Stata Corp. 2015). Le test de $\mathrm{Chi}^{2}$ de Pearson a été employé pour comparer les proportions des larves dans les différents gîtes. Le seuil de signification utilisé est de $5 \%$. 
Tia et al. J. Appl. Biosci. 2016 Étude du niveau de production larvaire d'Anopheles gambiae s.l. dans différents types de gîtes à Oussou-yaokro au Centre-Ouest et à Korhogo Côte d'Ivoire.

\section{RESULTATS}

Nature, localisation et dynamique des gîtes: $A$ Oussou-yaokro, 41 gîtes ont été prospectés. Ces gîtes peuvent être repartis en $6(15 \%)$ gîtes classiques (mares temporaires, rizières) et en 35 (85\%) gîtes atypiques (citernes ou jarres de conservation d'eau, barriques et abreuvoirs d'animaux) $\left(X^{2}=6,03, p=0,014\right)$ (Figure 2a, $2 b$ et $2 c)$. En ce qui concerne les gites atypiques, on note par ordre d'importance les citernes ou jarres de conservation d'eau ( $26 \%)$, les abreuvoirs d'animaux, et les barriques. On constate qu'il y a plus de gites atypiques que de gîtes classiques. En ce qui concerne la dynamique des gîtes, on a observé plus de gîte en saison pluvieuse (24 gîtes soit $59 \%$ ) qu'en saison sèche (17 gîtes, soit $41 \%$ ), mais cette différence n'était pas statistiquement significative $\left(X^{2}=1,10 ; p=0,294\right)$. Le tableau 1 montre la répartition et la dynamique des gîtes larvaires en fonction des quartiers et des types de gîtes à Korhogo. A Korhogo, au total 44 gîtes d'An. gambiae s.l. ont été enregistrés dont $91 \%$ de gîtes habituels ou classiques et $9 \%$ de gîtes inhabituels ou atypiques. Les gîtes habituels (40) étaient représentés par ordre d'importance de $48 \%$ de flaques routières, $18 \%$ de bas- fonds maraîchers, $11 \%$ de casiers rizicoles, $7 \%$ de mares temporaires, $5 \%$ de puits d'arrosage des maraîchers et $2 \%$ de trous d'empreint de terre. Les gîtes inhabituels comprenaient $5 \%$ de jarres d'eau résiduelle de beurre de karité, $2 \%$ de fosses septiques (Figure $2 d$ ) et $2 \%$ de pneus usagés (Figure 2e); ils contiennent en général de l'eau polluée riches en matières organiques. Tous ces gîtes sont occasionnés par les activités humaines, ce sont des gîtes anthropiques. II y a à Korhogo, plus de gites classiques que de gîtes atypiques. Concernant la dynamique des gîtes, en saison des pluies 34 gîtes $(77 \%)$ ont été enregistrés contre $10(23 \%)$ en saison sèche $\left(X^{2}=\right.$ $8,80 ; p=0,003)$. Quant à la localisation de ces gîtes, les quartiers insalubres abritaient $71 \%$ des gîtes d'An. gambiae s.l. contre $29 \%$ dans les quartiers salubres. Sur les 40 gîtes habituels rencontrés au cours des deux saisons, $75 \%$ se retrouvaient dans les quartiers insalubres tandis que $25 \%$ étaient dans les quartiers salubres $\left(X^{2}=7,27 ; p=0,007\right)$. Les gîtes inhabituels (atypiques) sont rencontrés dans les mêmes proportions aussi bien dans les quartiers insalubres que dans les quartiers salubres (2 gîtes de part et d'autres).

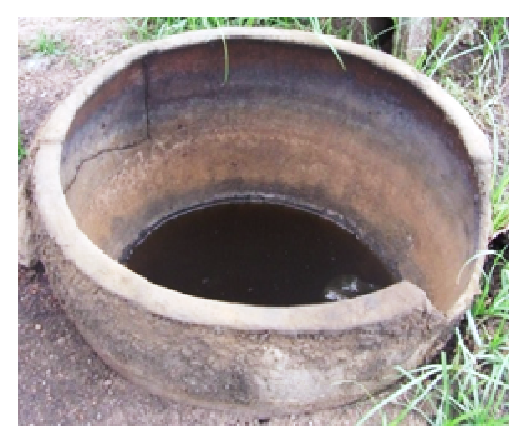

a : Jarre d'eau résiduelle de beurre de karité à Natio

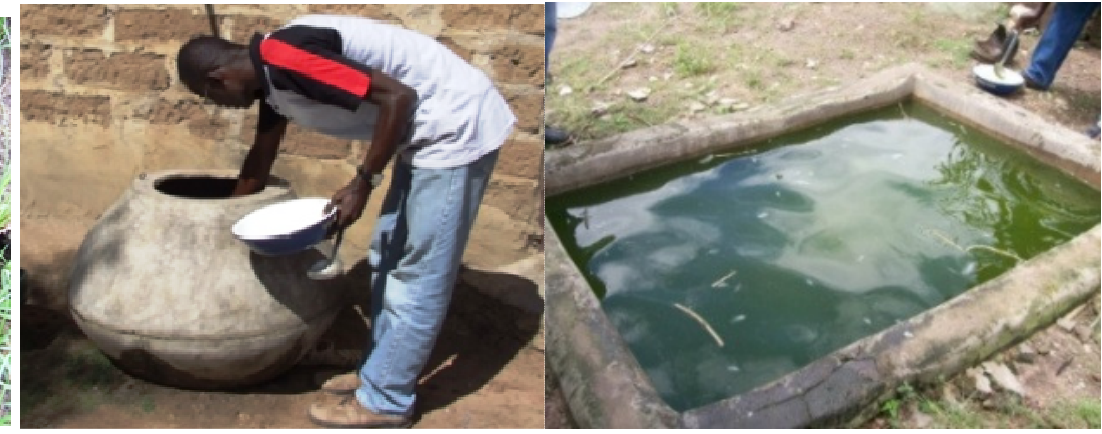

b : jarre de conservation d'eau (Oussou-yaokro) c : Abreuvoir d'animaux (Oussou-yaokro)

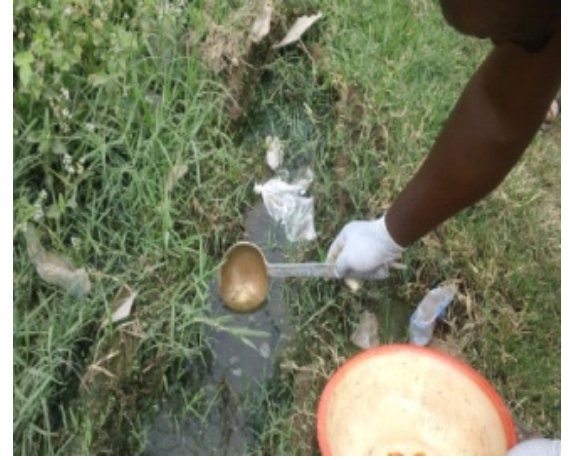

d: Fosse septique positif à $A n$. gambaie s.l. à Dem (Korhogo)

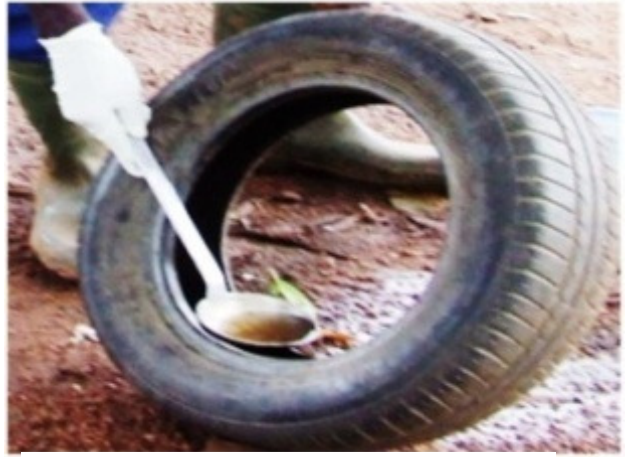

e: Pneu usagé au quartier Zone Industrielle (Korhogo) 
Tia et al. J. Appl. Biosci. 2016 Étude du niveau de production larvaire d'Anopheles gambiae s.l. dans différents types de gîtes à Oussou-yaokro au Centre-Ouest et à Korhogo Côte d'Ivoire.

\begin{tabular}{|c|c|c|c|c|c|c|c|c|c|c|c|c|c|c|c|c|c|c|c|c|}
\hline & \multirow{3}{*}{$\begin{array}{l}\text { Saisons } \\
\text { Type de quartier } \\
\text { Quartiers }\end{array}$} & \multicolumn{9}{|c|}{ Pluvieuse } & \multicolumn{10}{|c|}{ Sèche } \\
\hline & & \multicolumn{4}{|c|}{ Quartiers insalubres } & \multicolumn{4}{|c|}{ Quartiers salubres } & \multirow[b]{2}{*}{$\begin{array}{c}\text { Total A } \\
\mathrm{n}(\%)\end{array}$} & \multicolumn{4}{|c|}{ Quartiers insalubres } & \multicolumn{4}{|c|}{ Quartiers salubres } & \multirow[b]{2}{*}{$\begin{array}{c}\text { Total B } \\
\mathrm{n}(\%)\end{array}$} & \multirow[b]{2}{*}{$\begin{array}{c}\text { TOTAL } \\
\mathrm{n}(\%)\end{array}$} \\
\hline & & Ccdy & Natio & Dem & Total 1 & Mgaha & Ksrimé & $\mathrm{Zl}$ & Total 2 & & Ccdy & Natio & Dem & Total 3 & Mgaha & Ksrimé & $\mathrm{ZI}$ & Total 4 & & \\
\hline Gîtes & Flaques & 15 & 0 & 1 & 16 & 2 & 2 & 0 & 4 & $20(59)$ & 0 & 0 & 1 & 1 & 0 & 0 & 0 & 0 & $1(10)$ & $21(48)$ \\
\hline \multirow[t]{6}{*}{ Habituels } & Bas-fonds & 2 & 0 & 0 & 2 & 2 & 0 & 0 & 2 & $4(12)$ & 3 & 1 & 0 & 4 & 0 & 0 & 0 & 0 & $4(40)$ & $8(18)$ \\
\hline & Casiers rizicoles & 0 & 1 & 2 & 3 & 0 & 2 & 0 & 2 & $5(15)$ & 0 & 0 & 0 & 0 & 0 & 0 & 0 & 0 & $0(0)$ & $5(11)$ \\
\hline & Mares Temporaire & 0 & 0 & 0 & 0 & 1 & 1 & 0 & 2 & $2(6)$ & 0 & 1 & 0 & 1 & 0 & 0 & 0 & 0 & $1(10)$ & $3(7)$ \\
\hline & Trou d'empreinte terre & 0 & 1 & 0 & 1 & 0 & 0 & 0 & 0 & $1(2)$ & 0 & 0 & 0 & 0 & 0 & 0 & 0 & 0 & $0(0)$ & $1(2)$ \\
\hline & Puits d'arrosage & 0 & 0 & 0 & 0 & 0 & 0 & 0 & 0 & $0(0)$ & 1 & 1 & 0 & 2 & 0 & 0 & 0 & 0 & $2(20)$ & $2(5)$ \\
\hline & Total 1 & 17 & 2 & 3 & 22 & 5 & 5 & 0 & 10 & $32(94)$ & 4 & 3 & 1 & 8 & 0 & 0 & 0 & 0 & $8(80)$ & $40(91)$ \\
\hline Gîtes & Jarre de karité & 0 & 2 & 0 & 2 & 0 & 0 & 0 & 0 & $2(6)$ & 0 & 0 & 0 & 0 & 0 & 0 & 0 & 0 & $0(0)$ & $2(5)$ \\
\hline \multirow[t]{4}{*}{ inhabituels } & Fosses septiques & 0 & 0 & 0 & 0 & 0 & 0 & 0 & 0 & $0(0)$ & 0 & 0 & 1 & 1 & 0 & 0 & 0 & 0 & $1(10)$ & $1(2)$ \\
\hline & Pneus usagés & 0 & 0 & 0 & 0 & 0 & 0 & 0 & 0 & $0(0)$ & 0 & 0 & 0 & 0 & 0 & 0 & 1 & 1 & $1(10)$ & $1(2)$ \\
\hline & Total 2 & 0 & 2 & 0 & 2 & 0 & 0 & 0 & 0 & $2(6)$ & 0 & 0 & 1 & 1 & 0 & 0 & 1 & 1 & $2(20)$ & $4(9)$ \\
\hline & TOTAL & 17 & 4 & 3 & 24 & 5 & 5 & 0 & 10 & $34(100)$ & 4 & 3 & 2 & 9 & 0 & 0 & 1 & 1 & $10(100)$ & $44(100)$ \\
\hline
\end{tabular}

Ccdy : Cocody, Natio : Natiokobadara, Mgaha: Mongaha, Ksrimé: Kassirimé, Zl : Zone industrielle 

différents types de gîtes à Oussou-yaokro au Centre-Ouest et à Korhogo Côte d'Ivoire.

Propriétés physico-chimiques des gîtes positifs d'An. gambiae s.l. :Les propriétés-physico-chimiques des gîtes d'An. gambiae s.l. étudiés sont reportées dans le tableau 2. On observe que les gîtes atypiques (Jarres, flaques polluées et fosses septiques) d'An. gambiae s.l ont soit un $\mathrm{pH}$ acide avec une tendance neutre $(6,96)$, soit un $\mathrm{pH}$ basique au niveau des flaques d'eau polluées et des fosses septiques avec un maximum de 7,96 (saison pluvieuse). Ces gîtes ont également un taux d'oxygène, soit élevée $(98,43)$, soit faible $(2,35)$ saison sèche.
Concernant le taux de conductivité, il est tantôt élevé, atteignant $1337 \mu \mathrm{s} / \mathrm{cm}$ ou tantôt faible $(75,87$ $\mu \mathrm{s} / \mathrm{cm})$. Quant à la température, elle varie de 26 à $32^{\circ} \mathrm{C}$. En ce qui concerne les gîtes classiques (Cassiers maraîchers), ils ont une conductivité $(369,93 \mu \mathrm{s} / \mathrm{cm})$ proche de celle des gîtes atypiques, un $\mathrm{pH}$ basique $(7,12)$, une température de $31,5^{\circ} \mathrm{C}$ et un taux d'oxygène dissous de l'ordre de $46.43 \mathrm{mgl}^{-1}$. Les larves d'An. gambiae s.l. se développent donc dans plusieurs types gîtes de conditions physico-chimiques très variées.

Tableau 2 : Nature et caractéristiques physico- chimiques des gîtes positifs d'An. gambiae s.l. à Korhogo Nord Côte d'Ivoire, 2014/2015

\begin{tabular}{|c|c|c|c|c|c|c|c|c|c|c|c|}
\hline \multirow{2}{*}{$\begin{array}{l}\text { Saison } \\
\text { Paramètres } \\
\text { Type de gîtes }\end{array}$} & \multicolumn{5}{|c|}{ Pluvieuse } & \multicolumn{6}{|c|}{ Sèche } \\
\hline & $\mathrm{pH}$ & $\begin{array}{c}\mathrm{OD} \\
\left(\mathrm{mgl}^{-1}\right)\end{array}$ & $\begin{array}{l}\text { Cond. }(\mu \\
\mathrm{S} / \mathrm{cm})\end{array}$ & Sal & $\mathrm{T}^{\circ}\left({ }^{\circ} \mathrm{C}\right)$ & $\mathrm{pH}$ & $\begin{array}{c}\mathrm{OD}(\mathrm{mgl}- \\
1)\end{array}$ & $\begin{array}{l}\text { Cond. }(\mu \\
S / \mathrm{cm})\end{array}$ & Sal & $\mathrm{T}^{\circ}\left({ }^{\circ} \mathrm{C}\right)$ & Quartiers \\
\hline Jarres & 6,96 & 98,43 & 378,33 & 0,10 & 26,8 & 6,74 & 2,45 & 897,5 & 0,43 & 29,80 & Natio \\
\hline $\begin{array}{l}\text { Flaques } \\
\text { polluées }\end{array}$ & 7,78 & 48,55 & 382,67 & 0,16 & 28,95 & 7,73 & 6,68 & 75,87 & 1,17 & 32,07 & Cocody \\
\hline $\begin{array}{l}\text { C. } \\
\text { maraîchers }\end{array}$ & 7,12 & 46,43 & 369,93 & 0,20 & 31,5 & - & - & - & - & - & Mongaha \\
\hline $\begin{array}{l}\text { Fosses } \\
\text { septiques }\end{array}$ & 7,96 & 65,00 & 102,20 & 0,00 & 29,6 & 7,35 & 2,35 & 1337 & 0,65 & 31,75 & Dem \\
\hline
\end{tabular}

$\mathrm{T}^{\circ}$ : température, OD : oxygène dissous, Cond. : Conductivité, Sal : salinité, Natio : Natiokobadara, C. maraîchers : Casiers maraîchers

Production larvaire dans les types de gîtes: A Oussou-yaokro, 101 larves d'An. gambiae s.l. ont été récoltées dont $38(37 \%)$ dans les rizières, $26(26 \%)$ dans les citernes ou jarres, $15(15 \%)$ dans les abreuvoirs d'animaux (poulets et canards), 17(17\%) dans les mares temporaires et $5(5 \%)$ dans les barriques. En ce qui concerne la production larvaire des gites atypiques, on note par ordre d'importance, les citernes ou jarres de conservation d'eau, les abreuvoirs d'animaux et les barriques. Les gîtes atypiques et les gîtes classiques produisent respectivement $46 \%$ et $54 \%$ des larves collectées. Les larves d'An. gambiae s.l. colonisent de façon similaire les gîtes classiques (mares temporaires, rizières et bas-fonds) et les gîtes atypiques (citernes, barriques et abreuvoirs d'animaux $\left(X^{2}=3,36 ; p=0,066\right)$ (Tableau 3). A Korhogo, au total 3067 larves d'An.gambiae s.l .ont été récoltées dont 3034 (99\%) dans des gîtes habituels et $33(1 \%)$ dans les gîtes inhabituels ou atypiques. En tenant compte du facteur de salubrité, les gites des quartiers salubres produisent plus de larves que ceux des quartiers insalubres soit respectivement $2150(70 \%)$ larves et $917(30 \%)$ larves, et la différence est statistiquement significative $\left(X^{2}=19,51 ; p=0,00001\right)$. Au total, les productions larvaires sont plus abondantes dans les gîtes habituels et dans les quartiers salubres (Tableau 4). 
Tia et al. J. Appl. Biosci. 2016 Étude du niveau de production larvaire d'Anopheles gambiae s.l. dans différents types de gîtes à Oussou-yaokro au Centre-Ouest et à Korhogo Côte d'Ivoire.

Tableau 3: Production larvaire des différents types de gîtes à Oussou-yaokro, Centre-Ouest, Côte d'Ivoire, 2011/2012

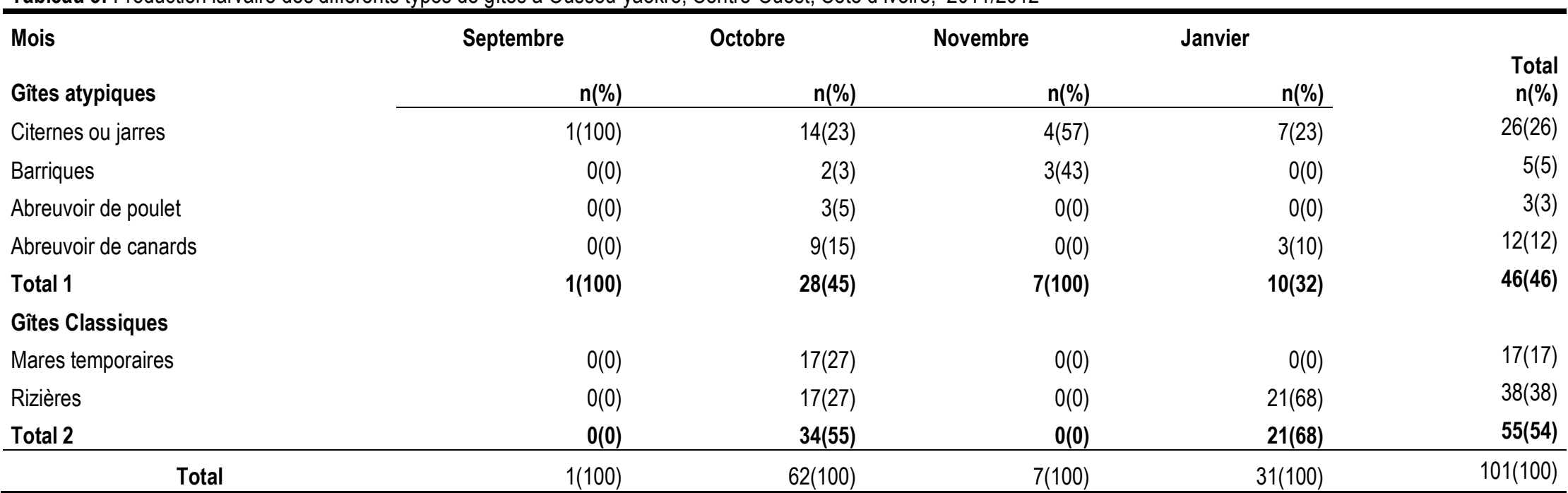

Tableau 4: Effectif des larves d'An. gambiae s.I. dans les gîtes positifs à Korhogo, 2014/2015

\begin{tabular}{|c|c|c|c|c|c|c|c|c|c|c|c|c|c|c|c|c|c|c|c|c|}
\hline \multirow{3}{*}{$\begin{array}{l}\text { Saison } \\
\text { Types de gîtes } \\
\text { Quartiers }\end{array}$} & \multicolumn{10}{|c|}{ Pluvieuse } & \multicolumn{10}{|c|}{ Sèche } \\
\hline & \multirow[b]{2}{*}{$\mathrm{CR}$} & \multirow[b]{2}{*}{ TET } & \multicolumn{3}{|c|}{ Habituels } & \multirow[b]{2}{*}{ S/total } & \multicolumn{3}{|c|}{ Inhabituels } & \multirow[b]{2}{*}{ Total 1} & \multicolumn{5}{|c|}{ Habituels } & \multicolumn{3}{|c|}{ Inhabituels } & \multirow[b]{2}{*}{ Total 2} & \multirow[b]{2}{*}{ TOTAL } \\
\hline & & & MT & $\mathrm{FL}$ & $\mathrm{CM}$ & & FRP & JUK & S/total & & MT & $\mathrm{CM}$ & PA & $\mathrm{FL}$ & S/total & FS & PU & S/total & & \\
\hline Natiokobadara & 17 & 7 & 0 & 0 & 0 & 24 & 0 & 5 & 5 & 29 & 119 & 9 & 3 & 0 & 131 & 0 & 0 & 0 & 131 & 160 \\
\hline Cocody & 0 & 0 & 0 & 947 & 11 & 958 & 13 & 0 & 13 & 971 & 0 & 254 & 0 & 0 & 254 & 0 & 0 & 0 & 254 & 1225 \\
\hline Mongaha & 0 & 0 & 1 & 117 & 1059 & 1177 & 0 & 0 & 0 & 1177 & 0 & 0 & 0 & 0 & 0 & 0 & 0 & 0 & 0 & 1177 \\
\hline Kassirimé & 408 & 0 & 40 & 23 & 0 & 471 & 0 & 0 & 0 & 471 & 0 & 0 & 0 & 0 & 0 & 0 & 0 & 0 & 0 & 471 \\
\hline Dem & 0 & 0 & 0 & 2 & 0 & 2 & 0 & 0 & 0 & 2 & 0 & 0 & 0 & 17 & 17 & 13 & 0 & 13 & 30 & 32 \\
\hline Zone industrielle & 0 & 0 & 0 & 0 & 0 & 0 & 0 & 0 & 0 & 0 & 0 & 0 & 0 & 0 & 0 & 0 & 2 & 2 & 2 & 2 \\
\hline TOTAL n(\%) & 425 & 7 & 41 & 1089 & 1070 & 2632(99) & 13 & 5 & $18(1)$ & $2650(100)$ & 119 & 263 & 3 & 17 & 402(96) & 13 & 2 & $15(4)$ & $417(100)$ & 3067 \\
\hline
\end{tabular}

$\mathrm{CR}=$ Casier rizicole, $\mathrm{TET}=$ Trou d'empreint de terre, $\mathrm{FL}=$ Flaques, $\mathrm{MT}=$ =Mares temporaires, $\mathrm{CM}=$ Casier maraîcher, $\mathrm{FRP}=$ Flaques routières polluées d'ordures ménagères, JUK = Jarres usagées de beurre de Karité, PA=Puits d'arrosage, FS=Fosse septique, PU=Pneus usagés. Total larves des gîtes habituels : 3034 (99\%), Total larves des gîtes inhabituels : 33 (1\%) 

différents types de gîtes à Oussou-yaokro au Centre-Ouest et à Korhogo Côte d'Ivoire.

Dynamique de la population larvaire d'An. gambiae s.l. : Dans le village de Oussou-yaokro, au total 101 larves d'An. gambiae s.l. Ont été récoltées, avec $63 \%$ en saison des pluies (Septembre et Octobre), contre $37 \%$ en saison sèche (Novembre et Janvier), tableau(3). En outre, la population larvaire d'An. gambiae s.l. a connu son pic au cours du moi d'Octobre (saison pluvieuse) et son faible taux en janvier (en saison sèche). De manière générale, la dynamique des populations larvaires suit relativement celle de la pluviométrie et en est donc liée (Figure 3). En saison des pluies, la plupart des gîtes larvaires sont positifs, mais en saison sèche (Janvier), seuls les citernes ou jarres, les barriques et les rizières produisent des larves. Seules les jarres de conservation d'eau (gîtes atypiques) ont une production larvaire régulière repartie sur toute la durée de l'étude. (aussi bien en saison qu'en saison des pluies) ; ces gîtes sont suivis des rizières, abreuvoirs et les barriques, enregistrés positifs deux mois sur quatre (octobre et janvier) en saison sèche et en saison des pluies. Les autres gîtes ont été enregistrés positifs pendant un seul mois (octobre) en saison des pluies (Figure 4).

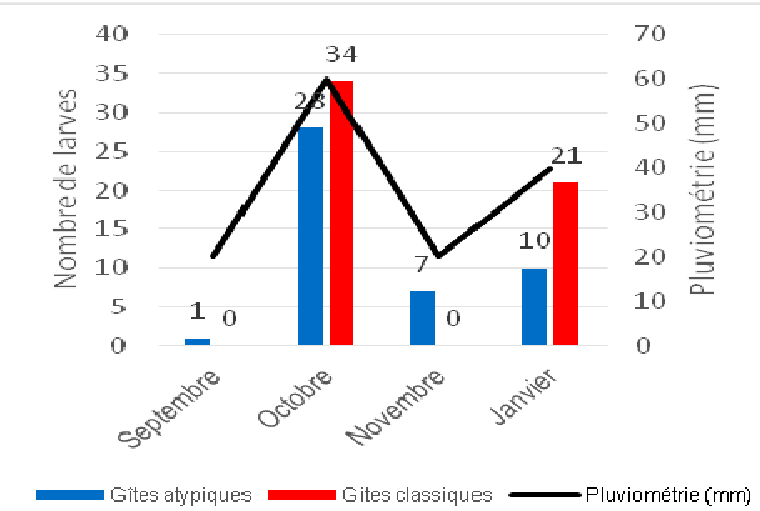

Figure 3 : Dynamique des populations larvaires d'An. gambiae s.l. dans les différents types de gîtes en fonction de la pluviométrie à Oussou- yaokro, Centre-Ouest, Côte d'Ivoire

A Korhogo, concernant la production saisonnière, 2650 $(86,5 \%)$ larves ont été récoltées en saison des pluies contre $417(13,5 \%)$ en saison sèche $\left(X^{2}=19,51 ; p=\right.$ 0,00001 ). Les gites habituels produisent plus de larves en saison des pluies (2639 ou 87\%) qu'en saison sèche (402 ou $13 \%$ ) tandis que les gîtes inhabituels produisent les larves au cours des deux saisons (18 en saison des pluies ou $56 \%$ et 15 en saison sèche ou $44 \%$ ) avec la même abondance $\left(X^{2}=2,21, p=0,51\right)$.

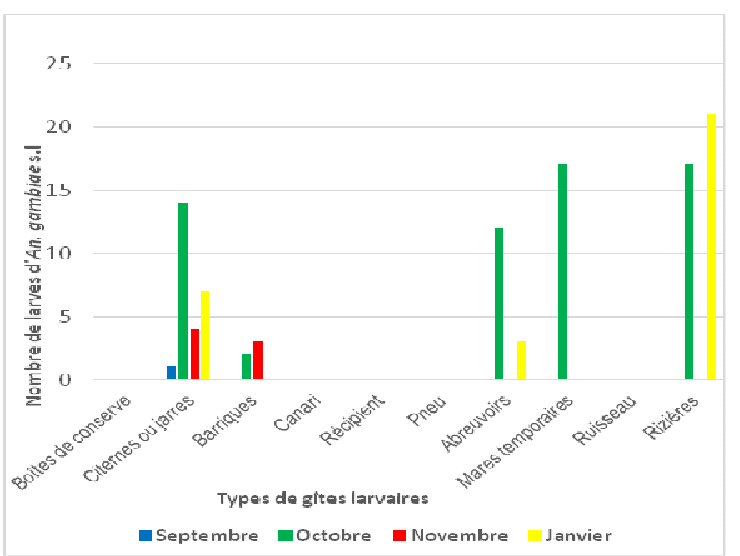

Figure 4 : Dynamique de la production larvaire d'An. gambiae s.l. dans chaque type gîtes à Oussou-yaokro, Centre-Ouest, Côte d'Ivoire 

différents types de gîtes à Oussou-yaokro au Centre-Ouest et à Korhogo Côte d'Ivoire.

\section{DISCUSSION}

Les déterminants de la transmission du paludisme comprennent entre autres la présence et la productivité des gîtes de reproduction des vecteurs de l'agent pathogène. Les prospections larvaires ont montré que les sites d'étude présentent une variété de gîtes larvaires. La plupart de ces gîtes sont anthropiques et sont situés dans le voisinage immédiat des populations tel qu'observé aussi au Bénin par Akogbeto(2000). Cette observation met en exergue la responsabilité des populations dans la pullulation des vecteurs du paludisme à travers la création des gîtes de ceux-ci. Aussi, est-il urgent d'accentuer la sensibilisation de ces populations à assainir leur cadre de vie par la suppression de ces gîtes. Nature des gîtes : Les gîtes recensés peuvent être répartis en gîtes classiques ou habituels et en gîtes atypiques ou inhabituels. Les gîtes classiques sont des points d'eau ensoleillés, propres, claires (Mouchet et al., 2004). Les gites atypiques sont des points d'eau relativement polluées, riches en matières organiques. En ce qui concerne les gîtes classiques, il s'agit à Korhogo, de flaques d'eau, de mares temporaires, de trous d'empreint de terre. Ces gîtes témoignent d'un assainissement défaillant du cadre de vie dû au laxisme des services publics compétents et/ou à la négligence de la population locale. De même, l'observation des casiers rizicoles, des bas-fonds exploités à la culture maraîchères et des puits d'arrosage montre le dynamisme de l'agriculture urbaine dans la localité, cause du paludisme urbain de même importance que le paludisme rural plus classique. Aussi, faut-il en prendre conscience et mener une véritable campagne de prévention et de soins antipaludiques au profit des riverains et de promotion et distribution gratuite de moustiquaire imprégnée à longue durée d'action (MILDA). A Oussou-yaokro, en milieu rural, les gîtes classiques observés sont les bas-fonds qui ceinturent le village et exploités à la riziculture et dont l'eau est conservée dans les jarres pour un usage domestique. Outre ces bas-fonds, la présence de nombreuses mares temporaires ou de flaques d'origine anthropique constituent une source de nuisance culicidienne pour les populations et un facteur de risque de paludisme dans cette localité. II faut y mener une sensibilisation à l'assainissement du cadre de vie et d'utilisation des MILDA. Concernant les gîtes atypiques ou inhabituels à Oussou-yaokro, les citernes ou jarres de conservation d'eau domestique sont les gîtes les plus abondants $(26 \%)$. Cela s'explique par le fait que les habitants d'Oussou-yaokro ont l'habitude de conserver l'eau. En effet, l'eau courante leur étant presqu'inaccessible, elles conservent l'eau des bas-fonds, des puits, rizières et ruisseaux dans ces citernes pour un usage domestique planifiée (Gbalégba, 2012). La conservation de l'eau des pluies ou des sources susmentionnées permet en effet de réduire l'effort physique imposé aux femmes, celui de parcourir de longues distances pour satisfaire les besoins hydriques de la famille. Outre ces citernes, An. gambiae s.l. a été récoltée aussi dans des barils, des abreuvoirs d'animaux (canard, poulet) à eaux verdâtres et ombragées (Figure 2d). On n'observe que An. gambiae s.l. a été récoltée aussi bien et avec la même abondance dans les gîtes classiques que dans les gîtes atypiques où l'eau est généralement trouble (citerne, barrique,) ou polluée (abreuvoirs d'animaux) comme l'ont constaté (Karch et al., 1993 ; Coffinet et al., 2009 ; Noumi et al., 2012).Cette observation révèle que ces gîtes notamment les jarres de conservation d'eau domestique ne sont plus l'apanage d'Ae. aegypti, mais que la présence d'An.gambiae dans ces gîtes atypiques devrait désormais interpeller les responsables des programmes de lutte contre le paludisme afin qu'ils en tiennent compte dans les projets de lutte anti-vectorielle. A Korhogo(en milieu urbain), An. gambiae s.l. a été récoltée dans des gîtes atypiques à eau polluée et sale tels que les pneus usagés, les fosses septiques et eaux résiduelles de fabrication de beurre karité. Concernant les eaux résiduelles de karité, elles sont boueuses, acides, riche en matières organiques non biodégradables (Service, 1989; Ouattara, 2009) non temporaires et donc atypique pour cette espèce rencontrée classiquement dans des gîtes temporaires, ensoleillée et claire (Mouchet et al., 2004). Les résultats de l'étude des propriétés physicochimiques des gîtes, montrent que An. gambiaes.l.se rencontre dans une variété de gîtes à $\mathrm{pH}$ soit acide ou basique, à taux d'oxygène réduit et une conductivité faibles ou élevés et une température identique ou légèrement plus élevée que celle des gîtes classiques, ce qui confirme son adaptation à de nouveaux types de gîtes. Selon Agula et al. (2010) c'est la forme M d'An. gambiae s.l. qui cohabite en milieu urbain avec Culex quinquéfasciatus dans les eaux polluées riche en matières organiques; il en conclut que l'adaptation de $A n$. gambiae s.l. à l'environnement urbain et à de nouveaux types de gîtes, ce qui constitue une menace sérieuse capable d'impacter la santé humaine. Cette observation confirme l'ubiquité d'An. gambiae s.l. notée par plusieurs auteurs dont (Diarra et Toguebaye, 2003; OMS, 2003; Mouchet et al., 2004; Pierrat, 2010). En effet, ces auteurs ont montré que les gîtes domestiques notamment les réservoirs d'eau domestiques sont non seulement des 


\section{Tia et al. J. Appl. Biosci. 2016 Étude du niveau de production larvaire d'Anopheles gambiae s.l. dans différents types de gîtes à Oussou-yaokro au Centre-Ouest et à Korhogo Côte d'Ivoire.}

gîtes incontestables d'Aedes, vecteurs majeurs de fièvre jaune, mais aussi peuvent abriter des larves d'anophèle, vecteur du paludisme et qu'il existe pour ce moustique des gîtes préférentiels et des gîtes inhabituels ou atypiques, pollués (eaux de bain, de linge, et de vaisselles, les réservoirs d'eau domestiques). Par ailleurs, la colonisation de ces gîtes inhabituels n'est pas liée à l'absence de gîtes préférentiels. Pierrat (2010) note qu'An.gambiae s.l. est en constante évolution, ce qui permet son adaptation à la pollution urbaine. Cette ubiquité est due à l'adaptation d'An.gambiae s.l à une gamme élargie de situations écologiques en rapport avec les changements climatiques. Selon Coluzzi et al., (1979), An. gambiae s.l. est doté d'une incroyable plasticité génétique qui lui permet de s'adapter à une variabilité de conditions environnementales. Ce potentiel adaptatif serait en grande partie lié à la présence d'inversions chromosomiques polymorphes observées sur le chromosome.

Production larvaire des gîtes: En comparant la production de ces deux types de gîtes, on constate qu'en milieu rural (Oussou-yaokro), les deux types de gîtes ont une production similaire certainement du fait de l'homogénéité des compositions physico-chimiques de ces gîtes. En revanche, en milieu urbain (Korhogo), les gîtes habituels ont une production larvaire supérieure à celle des gîtes atypiques, cela est dû au nombre et au degré de pollutions de ces derniers. En tenant compte de la salubrité du milieu, la production larvaire d'An. gambiae s.l. est plus élevée dans les quartiers salubres que dans les quartiers insalubres. Cette observation s'explique par le fait que les eaux des quartiers insalubres sont en général polluées et donc relativement peu propices à la prolifération d'An. gambiae s.l. Toutefois, le risque d'accès palustre dans ces quartiers est réel du fait de l'adaptation de plus en plus fréquente d' An. gambiae s.l. à ces gîtes atypiques (fosses septiques, eaux polluées) (Awolola et al., 2007). A Abidjan par exemple, la probabilité de mourir entre 1 et 5 ans serait de 15 fois plus élevée dans l'habitat précaire que dans l'habitat de standing (Gueu, 1993). Selon l'OMS, près de la moitié des citadins d'Afrique, d'Asie et d'Amérique latine sont atteints d'une ou plusieurs maladies associées à un approvisionnement en eau ou bien à un assainissement inadéquat (WHO, 2000). Pour pallier cette situation, il faut une meilleure gestion de l'environnement sanitaire urbain à travers la mise en place d'un observatoire des paramètres environnementaux et d'une politique suivi d'assainissement du cadre de vie des populations locales. Ce qui permettra des prises de décisions efficaces dans le cadre de la politique de restructuration des quartiers précaires pour leur intégration dans le tissu urbain.

Localisation des gîtes positifs: Concernant la localisation des gîtes positifs, ils sont uniformément répartie dans l'espace en milieu rural tandis qu'en milieu urbain, à Korhogo ces gîtes sont plus abondants dans les quartiers insalubres que dans les quartiers salubres. Les quartiers insalubres (Natiokobadara, Dem et Cocody) sont caractérisés par la prolifération dans les rues des déversoirs d'eaux usées, la prolifération des flaques d'eau due à un système d'assainissement défectueux et/ou à déversement anarchique d'eau de ménage (lessive, vaisselle) par les populations locales. De plus, la promiscuité des habitations constitue un frein à un système d'assainissement adéquat au point où les retenues d'eau sont nombreuses (Diabagaté, 2009). Dans les quartiers salubres (Mongaha, Kassirimé et Zone industrielle) au contraire, le système d'évacuation de l'eau est bien fonctionnel et effectivement utilisé par les ménages pour la gestion des eaux usées; les eaux ne sont pas évacuées dans les rues. Le cadre de vie est assaini au point où il y a très peu de flaques d'eau. En outre, les fosses septiques sont bien couvertes.

Dynamique de la production larvaire: Concernant l'abondance du vecteur dans le temps, elle est synchrone à l'évolution de la pluviométrie. Cette observation montre que la dynamique des populations du vecteurs du paludisme à Oussou-yaokro est rythmée par la pluviométrie et le comportement des populations locales notamment leur méthode de conservation et de gestion de l'eau en fonction des saisons. Si en saison des pluies, la plupart des gîtes sont positifs, il en va autrement en saison sèche où seuls les citernes d'eau domestiques et les rizières produisent des larves ; cela suggère qu'en saison sèche, outre les rares pluies, la gestion de l'eau dans les citernes pour les besoins quotidiens influence la dynamique des populations vectrices. En effet, face à la rareté de l'eau en saison sèche, les populations se ravitaillent prioritairement en eau de la seule pompe hydraulique du village et en gardent dans les citernes plus d'une semaine pour la boisson et les besoins quotidiens. Ces réserves d'eau dans les citernes sont utilisées de manière parcimonieuse pour en bénéficier sur une période de plus de 3 à 7 jours. Cette période est suffisante pour la conduite du cycle biologique d'An.gambiae s.l. (Mouchet et al., 2004). La solution à cette situation est la multiplication des pompes hydrauliques dans les villages en lieu et place d'une seule pour éviter que les populations locales conservent l'eau plus longtemps dans les citernes. En outre, les petites jarres doivent être entièrement vidées une fois par 
semaine et il faut en gratter la surface intérieure pour éliminer les œufs de moustiques; elles doivent être dotées d'un couvercle rigide et bien ajusté, être recouverts avec une pièce de tissus ou de grillage. De cette façon, l'eau de pluie peut y pénétrer. Ces jarres doivent être traitées avec du Téméphos pour y éliminer éventuellement les larves de moustiques. A ces mesures, il faut ajouter, la sensibilisation des villageois sur les causes et la gravité du paludisme, la lutte anti larvaire en

\section{CONCLUSION}

La lutte larvicide contre les vecteurs du paludisme se focalise généralement sur les gîtes classiques connus du principal vecteur notamment les collections d'eau claires et ensoleillées. La présente étude indique qu'An. gambiae s.l. se développe aussi dans des gîtes atypiques, tels que les jarres, les abreuvoirs d'animaux, les eaux des fosses septiques, les eaux résiduelles de beurre de karité, initialement propices à la reproduction d'Aedes aegypti et de Culex quinquefasciatus, respectivement vecteurs de la fièvre jaune et des

\section{REMERCIEMENTS}

Nous remercions les Chefs des localités d'Oussou-yaokro et de Korhogo ainsi que les populations. Nos remerciements vont également à l'endroit des techniciens entomologistes, Mrs Koné Moussa et Koffi Bernard. Ce travail a bénéficié du financement du Centre d'Entomologie Médicale et Vétérinaire(CEMV) de I'Université Alassane Ouattara (Bouaké) et et du

\section{REFERENCES BIBLIOGRAPHIQUES}

Agula R, Simard F, Kamdeni C, Shields T, Glass GE, Garver LS, Dimopoulos G., 2010. Geneomewide analysis of transcriptomic divergence between laboratory colony and field Anophèles gambiae mosquitoes of the $M$ and $S$ molecular form. Insect Microbiology, (19): 695-705.

Akogbeto M, 2000. Impact des modifications de l'environnement et du degré de salinité des gîtes d'eau saumâtre sur la dynamique de population d'An. melas, vecteur du paludisme dans le milieu côtier lagunaire du Benin. Cahier d'étude de recherches francophones/Agriculture. Colloque international « Eau et Santé »,9 (5): 422-427.

ANADER (2010) Relevés pluviométriques de 2009 à 2010. Agence Nationale de Développement 3. Rural, Bouaflé, 2010, 4p

Anonyme, 2015. Fabrication traditionnelle du beurre de karité par des groupements de femmes en milieu urbain avec du Téméphos pour les gîtes non pollués et le méthyl-chlorpyriphos dans des gîtes à eau polluée en vue de réduire la densité anophélienne (Rozendaal, 1999). Selon Gazin et al., (1985), une réduction de 20 fois la population anophélienne entraîne une réduction du risque quotidien de transmission palustre de 7,3 fois en zone de transmission permanente comme c'est le cas à Oussou-yaokro.

filarioses. Cette observation suggère la destruction de toutes sortes de gîtes de moustiques dans la lutte larvicide eue égard à cette ubiquité d'An. gambiae s.l et une sensibilisation suivie des populations locales et des pouvoirs publiques à un assainissement du cadre de vie. Par ailleurs l'amélioration du système d'évacuation des eaux usées domestiques et la construction de pompes villageoises en nombre suffisant pourrait éviter aux populations de conserver l'eau sur une longue période dans des jarres.

programme spécial UNICEF/UNDP/Banque Mondiale/OMS de Recherche et de formation sur les Maladies Tropicales (TDR) en collaboration avec le Centre de Recherche pour le Développement International du Canada (CRDI), Dr Koné Brama, projet NB20283"

Afrique de l'Ouest [www.africa-shea-butter.com] consulté enligne le 29/01/2015.

Awolola TS, Oduola AO, Obansa JB, Chukwurar NJ, Unyimadu JP,2007.Anopheles gambiae s.S. breeding in polluted water bodies in urban Lagos, south-western Nigeria. J Vector Borne Dis., 44: 241-244.

Coffinet T, Rogier C, Pages F, 2009.Évaluation de l'agressivité des anophèles et du risque de transmission du paludisme: méthodes utilisées dans les Armées françaises. Méd. Trop., 69 : 109-122.

Coluzzi M, Sabatini A, Petrarca V, et Di Diego MA, 1979. Chromosomal differenciation and adaptation to human environnement in Anopheles gambiae complexe. Trans. R. Soc. Trop. Med. Hyg., 7: 483-497.

Diabagaté S, 2009. Analyse de la situation de l'assainissement dans les quartiers défavorisés 
d'Abidjan : Cas du quartier Sagbé dans la commune d'Abobo, Mémoire de DEA de Géographie option Environnement, IGT, Université d'Abidjan Cocody, 123p.

Fournet $F$, Cussac M, Ouari A, Meyer PE, Hyacinthe K, Toé HK, Gouagna LC, Dabiré RK. Diversity in anopheline larval habitats and adult composition during the dry and wet seasons in Ouagadougou (Burkina Faso). Malaria Journal. 2010; 9(78).

Gazin P, Robert V, Akogbeto M, Carnevale P, 1985. Réflexions sur les risques d'infection palustre selon la densité et l'infectivité anophélienne. Ann Soc Belge Méd Trop; (65) : 263-269.

Gbalégba NGC, 2012. Étude des facteurs de risques du paludisme à Oussou-yaokro, village de la Souspréfecture de Bouaflé, Côte d'Ivoire, Mémoire de $D E A, C E M V$, Université Alassane Ouattara,67p.

Girod R, et Le Goff G, 2006. Inventaire actualisé des moustiques (Diptera: Culicidae) des îlots français de Europa, Juan-de-Nova et GrandeGlorieuse (Canal du Mozambique, océan Indien). Bull. Soc. Pathol. Exot., (99), 2 :122128.

Gueu GD, 1993.Épidémiologie des maladies infectieuses transmises par voie hydrique à Abidjan. Thèse Pharmacie,Univ. Abidjan, 211p.

INS, 2014. Institut National de la Statistique [http://www.ins.ci/n]. Consulté en ligne le 20 février 2014

Karamoko D, et Sikina T, 2003. Répartition et évolution saisonnière des infections microsporidiennes chez les larves de moustiques de la région de Dakar, Sénégal, Sciences et Médecine, Série A, Vol 02, 2003.

Karch S, Asdi N, Manzambi ZM, Salaun JJ, 1993.La faune culicidienne et sa nuisance à Kinshasa (Zaîre). Bull., Soc., Pathol., Exot.,86: 68-75.

Koffi D, Touré OA, Varela ML, Womas IV, Béourou S, Brou S, Ehouman MF, Gnamien L, Richard V, Djaman JA, and Perraut R, 2015.Analysis of antibody profiles in symptomatic malaria in three sentinel sites of Ivory Coast by using multiplex, fluorescent, magnetic, bead-based serological assay (MAGPIX ${ }^{\mathrm{TM}}$ ). Malar J (2015) 14:509

Koudou BG, Tano Y, Doumbia M, Nsanzabana C, Cissé G, Girardin O, Dao D, N'Goran EK, Vounatsou P, Bordmann G, Keiser J, Tanner M, Utzinger J. 2005. Malaria transmission dynamics in central Côte d'Ivoire: the influence of changing patterns of irrigated rice agriculture. Med Vet Entomol. $2005 ; 19(1)$
Mouchet J, Carnevale P, Coosemans M, Julvez J, Manguin S, Richard-Lenoble D, et Sircoulon J, 2004. Biodiversité du paludisme dans le monde. Editions John Libbey Eurotext., Paris. 428 p.

Noumi ES, Dabat MH, et Blin J, 2012. Développement durable de la transformation du karité en valorisant énergiquement les résidus organiques, Ci Fem2012-Art : 21-39.

Ouattara A, 2009. Contribution à la mise en place d'un système de management environnemental et santé-sécurité dans les productions industrielles et artisanales du beurre de Karité. Cas de Sarepta SA, association Ragussi et cedivanenyée. Mémoire de fin de cycle, Master professionnel II en sciences du danger, Université de Ouagadougou, pp 67.

OMS, 1975.Manual of practical entomology in malaria, part II, 110p.

OMS, 2003. Entomologie du paludisme et de contrôle des vecteurs. Rapport du département du contrôle, de la prévention et de l'éradication. Groupe des maladies transmissibles. WHO/CDS/CPE/SMT/2003, 102p.

OMS, 2014. Entomologie du paludisme, formation à la lute antivectorielle. Guide du participant, 2014a, $180 \mathrm{p}$

OMS, 2014. World Malaria Report, World health Organization, Geneva, 2014b, 227 p

OMS, 2015. World Malaria Report, World health Organization, Geneva, 2015, $243 p$

Pierrat C, 2010. Des moustiques et des hommes : les territoires du paludisme à Tori-Bossito (sud du Bénin). PhD Thèse de Doctorat de Géographie, Université Paris I Panthéon-Sorbonne, 102p

Rozendaal JA, 1999. La lutte antivectorielle, méthodes à usage individuelle et communautaire, Doc OMS, $17 p$

Service MW,1989. Irrigation/boom or bane? In « Service, MW. Demography and vector-borne diseases ». CRC Press ed, Boca Raton, 283-301.

Stata Corp. 2015. Stata Statistical Software: Release 14. College Station, TX: Stata Corp LP

WHO,2000. Global water supply and Sanitation Assessment, World Health Organization, Geneva:2-9

Yebakima A, 1989. La lutte contre Aedes (Stegomyia) aegypti : des études entomologiques à l'éducation sanitaire, l'exemple de la Martinique. Maladies tropicales transmissibles. Ed. AUPELF-UREE John Libbey Eurotext. Paris, 4750. 